\title{
nary hypertension:
} how should our clinical practice reflect recent advances?

\author{
Mark Toshner
}

Affiliation: Pulmonary Vascular Diseases Unit, Papworth Hospital, Cambridge, UK.

Correspondence: Mark Toshner, Papworth Hospital, Papworth-Everard, Cambridgeshire, CB23 8RE, UK. E-mail: mrt34amedschl.cam.ac.uk

\section{$\circ$} @ERSpublications

The largest genetic screen to date shows that some pulmonary hypertension patients should be offered genetic testing http://ow.ly/WE1vG

In this issue of the European Respiratory Journal, GIRERD et al. [1] summarise their experience to date with genetic testing for reported mutations in cohorts of patients with familial pulmonary arterial hypertension (PAH), idiopathic PAH, pulmonary veno-occlusive disease (PVOD) and pulmonary capillary haemangiomatosis (PCH). This collective work from the French Referral Centre of Pulmonary Hypertension represents a current state of the art in a fast moving and complex area from a group with considerable experience. In the last decade, we have moved from a simple and unequivocal association of one gene; heterozygous germline mutations in the bone morphogenetic protein receptor type 2 (BMPR2) [2, 3], to multiple hits in the BMPR2 signalling pathway [4-8], to additional genes in non-canonical BMP pathways of uncertain clinical significance or definitively agreed frequencies [9-11]. How to test, what to test and how to counsel is already a potential minefield. It is only likely to become more complicated in the immediate future given the technological advances and rapidly decreasing costs involved in large-scale genetic studies. So for now, we have a well-delineated step-wise model set out, in which patients are first tested for BMPR2 and, if negative, a sub-selected population proceeded to activin receptor-like kinase (ALK)1 and endoglin testing. EIF2AK4 (eukaryotic translation initiation factor 2 alpha kinase 4) was only screened in patients suspected of having PVOD/PCH. This is not dissimilar to the recent change to guidelines where it is now recommend that genetic testing should be offered in $\mathrm{PVOD} / \mathrm{PCH}$, familial and sporadic $\mathrm{PAH}$, but largely leave the specifics of this to local guidelines and regulations. If BMPR2 testing is negative, it is suggested that endoglin/ALK1 should be considered for patients aged under 40 years or with a history (or family history) of hereditary haemorrhagic telangiectasia (HHT) [12]. In a French cohort, those with a familial history were also tested for SMAD9, KCNK3 and CAV-1of PAH if negative for BMPR2/ALK1/endoglin.

This pragmatic approach largely confirms the primacy of BMPR2 in both the heritable and idiopathic disease groups with prevalence in idiopathic $\mathrm{PAH}$ at around $15 \%$ and familial $\mathrm{PAH} 84 \%$, very much in line with previous reports $[13,14]$. In fact, it is notable how few of the other reported mutations have been found in this large cohort of 529 patients, though care needs to be taken as not all patients were screened for the full panel of genetic mutations and the researchers were selective in their filtering. There were nine ALK1 mutations and one endoglin mutation in the idiopathic cohort and only two KCNK3 mutations, in familial disease only, with a further three patients in the familial cohort having an ALK1 mutation. All of the positive ALK1 patients had clinical features or a family history of HHT. No mutations were found in SMAD9 or CAV-1.

BMPR2 therefore retains its place as the undisputed champion of genetic pathophysiology, although the testing criteria and algorithm somewhat stack the odds in its favour. In idiopathic PAH, there is clear importance in testing and counseling patients, but probably only in BMPR2 in the first instance as things

Received: Nov 092015 | Accepted after revision: Nov 262015

Conflict of interest: None declared.

Copyright OERS 2016 
stand. Of note they report equivocal evidence for changes in the BMPR2 promoter region. As these data are only in 10 patients, the jury remains out on the importance of the promoter region.

It is reassuring that clinical features or a family history were present in all ALK1 mutation carriers and clarifies the continued importance of taking a full history and accurate examination to target those patients who might need further testing. In familial disease, it looks with additional rare genetic mutation we are into the laws of diminishing returns, but a proportion of familial disease still remains unexplained by our current knowledge of the genes involved. It is likely that in the next 10 years we will be filling in the gaps with prospective whole genome sequencing now available and already in progress in large patient cohorts [15]. This should additionally clear up the question about how frequent some of the newer described genetic mutations are, and whether we should be changing our routine practice to incorporate some or all of them. For now, the French Referral Centre data gives us confidence that the current approach remains acceptable.

In PVOD/PCH, this work strongly solidifies the case for EIF2AK4 with sporadic cases now reported in $9 \%$ of the French cohort. To have built a patient cohort of 81 patients represents a significant achievement in a very rare disease, and really puts EIF2AK4 centre-stage. It is going to be difficult to amass enough patients to study but preliminary efforts to look at exercise physiology have been undertaken [16] and an intriguing link to drug-induced vasculopathy with mitomycin suggested [17]. The recapitulation of the mitomycin changes in an animal model also suggests some of the pathway pathophysiology may be traceable in animal models [17]. We can be confident that we have a new pathway of relevance to the pulmonary vascular field. Much as we have in the last 10 years needed to reassess basic pulmonary vascular science through the prism of BMPR2 across a wide spectrum of the causes of pulmonary hypertension, we now need to discern if this pathway is restricted in relevance to PVOD/ $\mathrm{PCH}$, or of more general importance.

The authors have also provided a nice summary of the mechanics of how this is all explained to the patients and their families including some data from a survey giving us some understanding of patient perceptions. This is particularly useful given the lack of published work in this area and the guidance, by necessity, referring to local regulations. Cultural and ethical considerations mean that it is difficult to apply a "one size fits all" algorithmic approach worldwide. Insight into how this is done in differing, experienced centres and networks is of definite value. Ultimately, whilst this will remain an area for imminent innovation and exploration, we still have to communicate the results to our patients and make sure the potential benefits and ethical issues raised by more complex genetic tests are not lost or forgotten.

\section{References}

1 Girerd B, Montani D, Jaïs X, et al. Genetic counselling in a national referral centre for pulmonary hypertension. Eur Respir J 2016; 47: 541-552.

2 Lane KB, Machado RD, Pauciulo MW, et al. Heterozygous germline mutations in BMPR2, encoding a TGF-beta receptor, cause familial primary pulmonary hypertension. Nat Genet 2000; 26: 81-84.

3 Thomson J, Machado R, Pauciulo M, et al. Familial and sporadic primary pulmonary hypertension is caused by BMPR2 gene mutations resulting in haploinsufficiency of the bone morphogenetic protein tuype II receptor. J Heart Lung Transplant 2001; 20: 149.

4 Trembath RC, Thomson JR, Machado RD, et al. Clinical and molecular genetic features of pulmonary hypertension in patients with hereditary hemorrhagic telangiectasia. N Engl J Med 2001; 345: 325-334.

5 Nasim MT, Ogo T, Ahmed M, et al. Molecular genetic characterization of SMAD signaling molecules in pulmonary arterial hypertension. Hum Mutat 2011; 32: 1385-1389.

6 Gallione CJ, Klaus DJ, Yeh EY, et al. Mutation and expression analysis of the endoglin gene in hereditary hemorrhagic telangiectasia reveals null alleles. Hum Mutat 1998; 11: 286-294.

7 Shintani M, Yagi H, Nakayama T, et al. A new nonsense mutation of SMAD8 associated with pulmonary arterial hypertension. J Med Genet 2009; 46: 331-337.

8 Boucherat $\mathrm{O}$, Chabot S, Antigny F, et al. Potassium channels in pulmonary arterial hypertension. Eur Respir J 2015; 46: 1167-1177.

9 Ma L, Roman-Campos D, Austin ED, et al. A novel channelopathy in pulmonary arterial hypertension. $N$ Engl $J$ Med 2013; 369: 351-361.

10 Austin ED, Ma L, LeDuc C, et al. Whole exome sequencing to identify a novel gene (caveolin-1) associated with human pulmonary arterial hypertension. Circ Cardiovasc Genet 2012; 5: 336-343.

11 Remillard CV, Tigno DD, Platoshyn O, et al. Function of Kv1.5 channels and genetic variations of KCNA5 in patients with idiopathic pulmonary arterial hypertension. Am J Physiol Cell Physiol 2007; 292: C1837-C1853.

12 Galie N, Humbert M, Vachiery JL, et al. 2015 ESC/ERS Guidelines for the diagnosis and treatment of pulmonary hypertension. Eur Respir J 2015; 46: 903-975.

13 Machado RD, Southgate L, Eichstaedt CA, et al. Pulmonary arterial hypertension: a current perspective on established and emerging molecular genetic defects. Hum Mutat 2015; 36: 1113-1127.

14 Larkin EK, Newman JH, Austin ED, et al. Longitudinal analysis casts doubt on the presence of genetic anticipation in heritable pulmonary arterial hypertension. Am J Respir Crit Care Med 2012; 186: 892-896.

15 National Cohort Study of Idiopathic and Heritable Pulmonary Arterial Hypertension. https://clinicaltrialsgov/ct2/ show/NCT01907295. Data last accessed: Dec 2016.

16 Laveneziana P, Montani D, Dorfmuller P, et al. Mechanisms of exertional dyspnoea in pulmonary veno-occlusive disease with EIF2AK4 mutations. Eur Respir J 2014; 44: 1069-1072.

17 Perros F, Gunther S, Ranchoux B, et al. Mitomycin-induced pulmonary veno-occlusive disease: evidence from human disease and animal models. Circulation 2015; 132: 834-847. 